\title{
Evaluating Perception, Characteristics and Research Directions for Internet of Things (IOT): An Investigational Survey
}

\author{
Bhavana A \\ Assistant Professor, NIE Institute of Technology \\ Mysore, Karnataka, India
}

\begin{abstract}
Internet of things (IoT) is considered to be one of the most recent emerging trends which connect variety of physical objects embedded with electronics equipment's for achieving a particular goal. Since 2013 the concept of IoT has been introduced in the field of networking for converging multiple devices and technologies. In the recent times many researchers have focused in the area of Internet of things as it is considered for handling multiple applications of many diverse fields. This paper focuses on collecting the information of an existing research trend of Internet of Things. The significant findings of this paper provide better resolution in the field of Internet of things which will be quite beneficial for future researchers, academician and Industries. Research gap and performance analysis of the existing studies considered as a major contribution of the proposed survey.
\end{abstract}

\section{Keywords}

Internet of Things, Context modelling, Sensor networks, Sensor Nodes.

\section{INTRODUCTION}

The internet is a global infrastructure of interlinked computing devices where different computing systems are connected worldwide for sharing information. Computing devices can be any type such as mobile devices, personal computers, laptops or the computers of various workstations. The internet is also known as network of networks, which concludes various private , public, academic, and business networks from local to global scope and surrounded by various resources like broad array of electronics, wireless and optical network technologies. The speed of the local machines varies when various nodes are connected to the internet as the connection depends on the Internet Service Providers [1]. Sometimes people also get confused between the words internet and the World Wide Web (WWW) but they are different in nature. The concept of internet says World Wide Web is a collection of various websites and various web pages that one can access with the help of internet where all the connected nodes work cooperatively for sharing and exchanging information [2]. Although Internet provides connectivity of various computing devices, but still there are many cases where the devices for collecting the data are not always computing. Such devices may be normal sensor nodes or mobile nodes or cellular phones. The present internet architecture doesn't support complete connectivity of the heterogeneous data collection devices for which purpose critical raw data collection and its transmission over internet is still a challenging task. Before discussing the internet of Things into depth it is advisable to have a generalized idea about the evaluation of internet. It has been observed that in the study of [3] in early 60's talks about the first possible communication and data transmission between two computing devices. In early 1980's the concept of TCP/IP protocol mechanisms has been introduced and after few years various applications of internet have successfully launched into the Global markets. The growth of the internet has become more popular as World Wide Web started in the year of 1991. Later based on the concept of Internet of Things (IoT) various mobile devices with distributed computing systems started communicating with each other and various trends towards development of social media have been introduced for connecting people globally [4]. The current research is trying to connect the various objects presented around us for ease of communication via internetworking. Fig 1 gives an overview about the various phases associated with the evaluation of internet. Since many years the concept of Internet of Things gained the significant attention of many computer scientists, researchers and academicians as well as industries. In the field of information and communication technology more and more applications are being embedded in various devices such as sensors for the ease of communication between various objects. The growth of ICT transforms the physical world into information and knowledge sharing based system which includes various objects of our physical world to be active for sharing information with other members belongs to a particular network that can be wired or wireless. It is often seen that the objects reside in the same network uses the same internet protocol, in that way a large amount of objects will be able to recognize various changes of parameters and events without any type of human involvement. The development of various applications in the field of ICT enables the expansion of interconnected things which will be using wireless and $5 \mathrm{G}$ network infrastructure in future. The internet of things enables various objects of virtual world to be connected with the physical environment where the mobile networks are deployed to fulfill the demands of 2550 billion things. In the recent times the Internet of things gained the attention of various researchers as it has the capabilities of offering a new wireless communication technology for various personal, home and business area networks for maintaining a large number of connections. Internet of things requires comprehensive systems approach including various intelligent applications for addressing the smart environment. The term Internet of things was firstly introduced in the paper [5] where the study mentioned how IoT can accelerate the development and growth in the field of information and communication technology (ICT). There are various definitions given by many researchers as IoT is considered as a very new concept of research and still in its early stages 


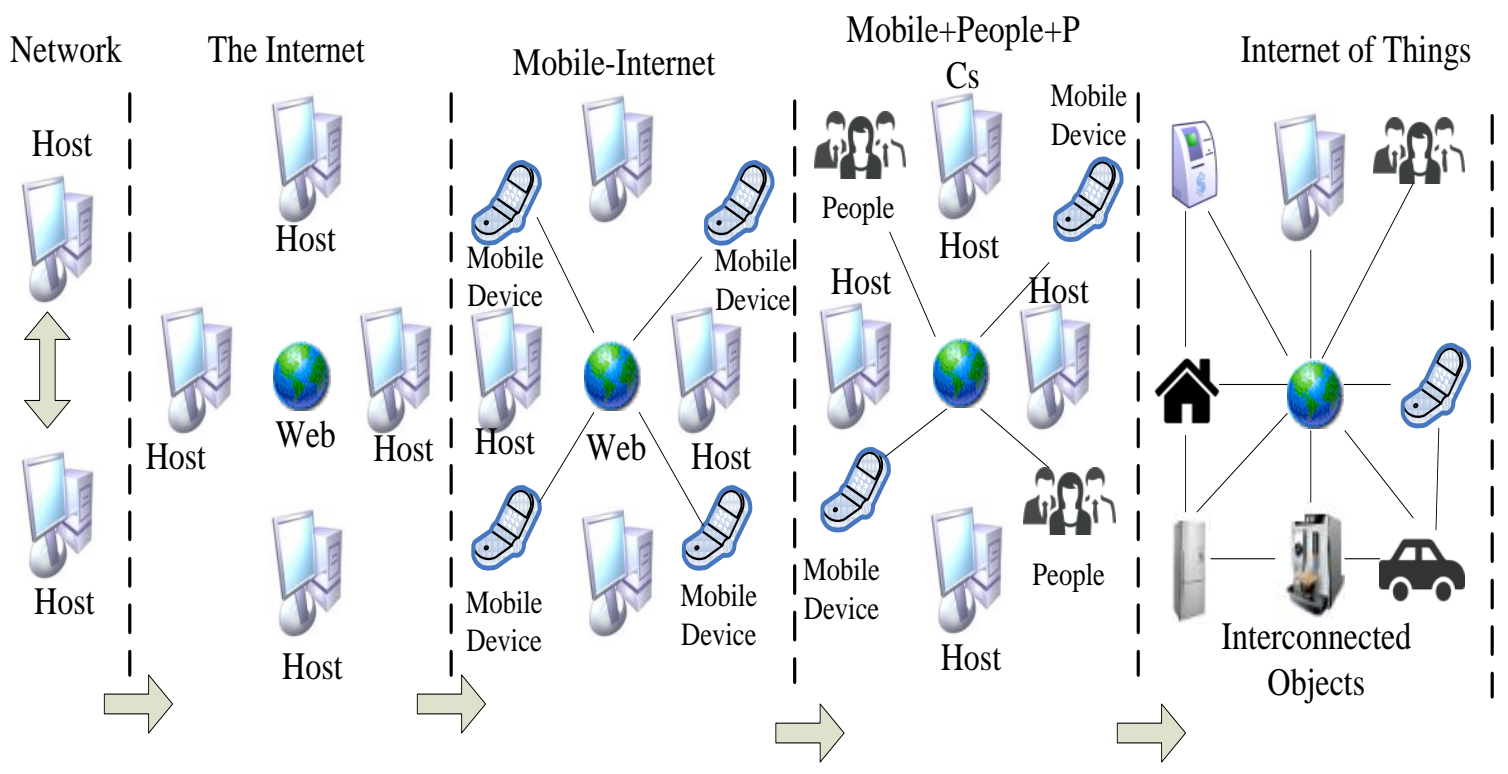

Fig.1. Evaluation of Internet

\subsection{Characteristics of the IoT}

It has been observed that there are most significant trends and technologies, which have an impact on Information Technology and services as well as Internet of Things (IoT) for the next five years (Fig.2). These things consist of specific IP addresses by which it can be tracked from anywhere. It is also found in the study of [6] that the internet is expanding day by day with huge amount of enterprise assets where most of the enterprises and technology vendors are not yet familiar with the fact of expanded internet and they are not operationally and organizationally ready. The four basic emerging usage models which have been found are

- Manage

- Monetize

- Operate

- Extended

These four properties can be utilized in the field of information, places, things and people consequently it is also applicable to so called Internet of Things. The use of IP network communication in the field of wireless sensor network opens the door for the convergence of large IToriented networks with real time applications.

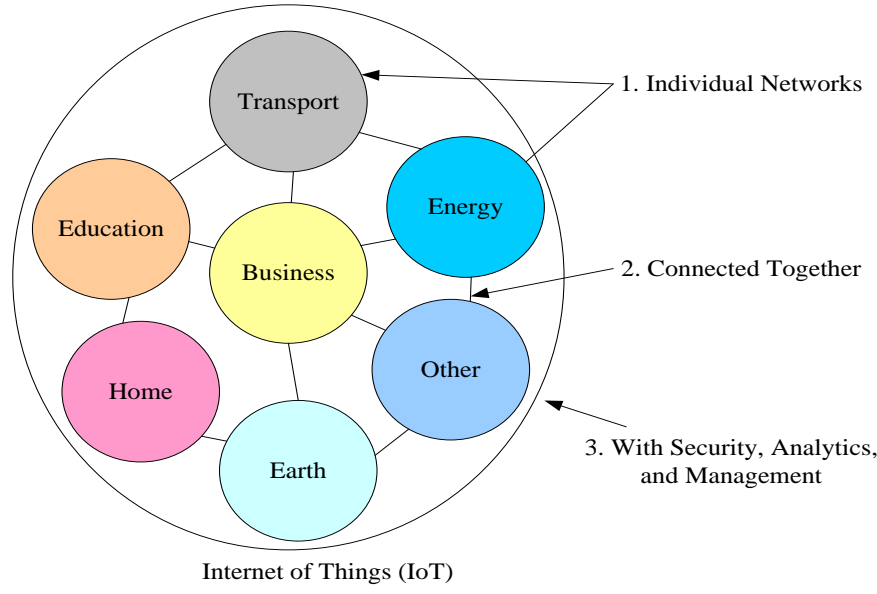

Fig.2. Internet of Things
The important characteristics of Internet of Things are described as follows:

- Interconnectivity: According to the concept of Internet of Things anything can be connected with the global communication and information infrastructure.

- Things-related Services: Various things related services such as privacy protection, semantic consistency between physical things and their associated virtual things can be provided by IoT

- Heterogeneity: The devices which are interconnected with the IoT infrastructure are heterogeneous in nature as they require different hardware platforms and network environment.

- Dynamic Challenges: The state of various devices which are present in the IoT can change dynamically for example sleeping/waking up, connected/disconnected etc. Moreover it uses the dynamic topology of the network.

- Enormous Scale: As the internet of things is not a single technology here various new technologies are connected and enabled. The enormous scale of Internet of Things talks about the higher order of magnitude associated with the various devices of IoT [7].

\subsection{Essential components of IoT}

This section provides a brief discussion about the sensor network which is the most essential component of IoT. A sensor network is a collection of one or more sensor nodes which communicates between each other and transmits data among each other to complete a particular task of that network. In the sensor network architecture it can be seen that all the sensors can be homogeneous or heterogeneous in nature and all the sensors uses different technologies and protocols [8]. The essential components of IoT includes usually sensor (both high and low end types) that perform data aggregation and forwards the aggregated data to the sink node, which are further transmitted to the high computational devices usually stored and accessed via cloud environment. The following Fig 3 shows the layer architecture of the sensor network. 


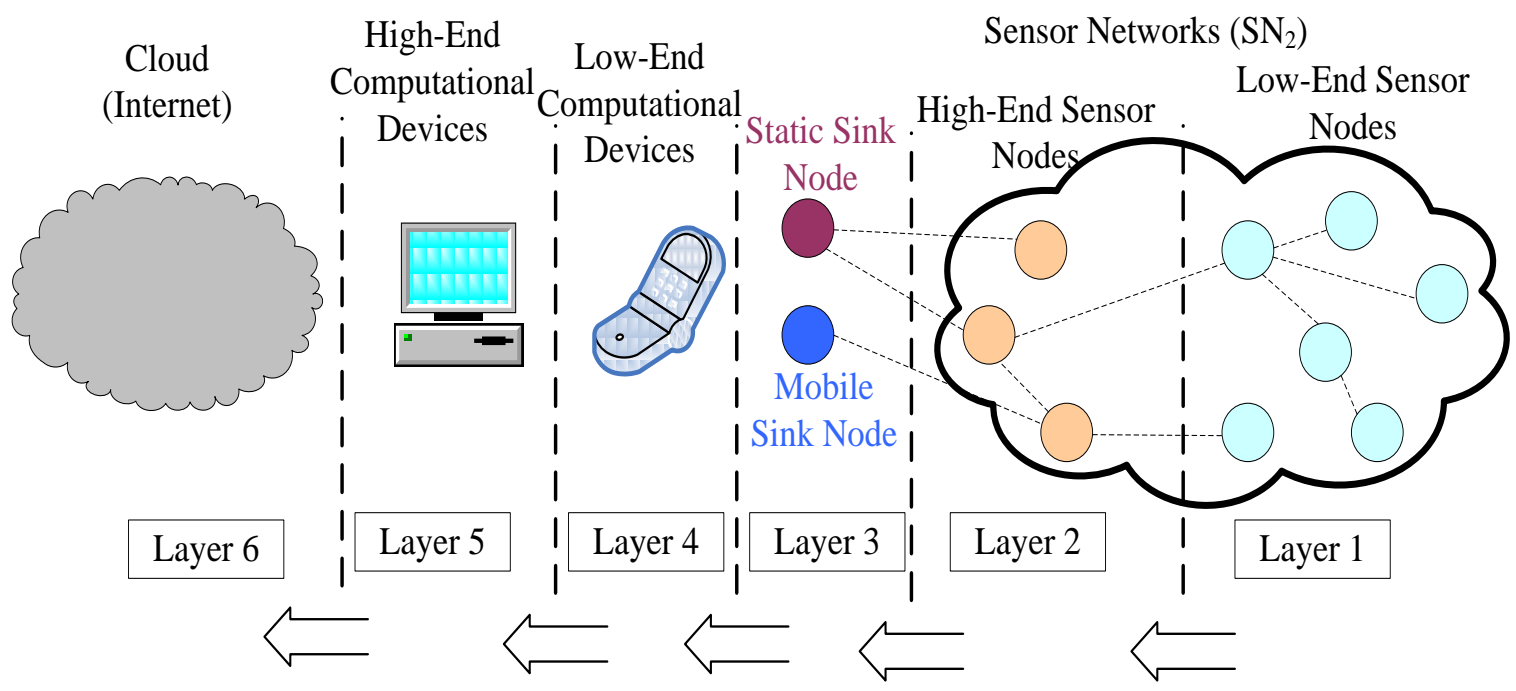

Fig.3. Various Components of Sensor Networks

\subsection{Relationship between IoT and Sensor Network}

As the previous discussed sections give an overview about the concept of IoT and Sensors. In this section the relationship between IoT and Sensors have been highlighted. As per the study of [7] IoT comprises sensors and actuators. The collection of data is done by sensors then it process the data and make decisions according to the decision actuator which is known as a motor for controlling various devices perform the decided action.

Sensor networks consist of the sensors and hardware, firmware and a thin layer of software framework where the IoT consists of many thing including sensor networks. In the
IoT various computational devices are activated with APIs and a software layer. Since many years sensor networks are designed and developed for some specific application purpose from now on some of the recent study says that it will be applicable in the field of IoT for general purpose such as some sensors will be deployed nearby a newly built building to sense its structural condition thus that will definitely reduce the labour work. It also have been found that the existence of IoT infrastructure is nothing without sensor network as the SN provides the majority of the hardware and infrastructure support (e.g. .Sensing and Communication). The following figure shows the relationship between IoT and Sensor networks.

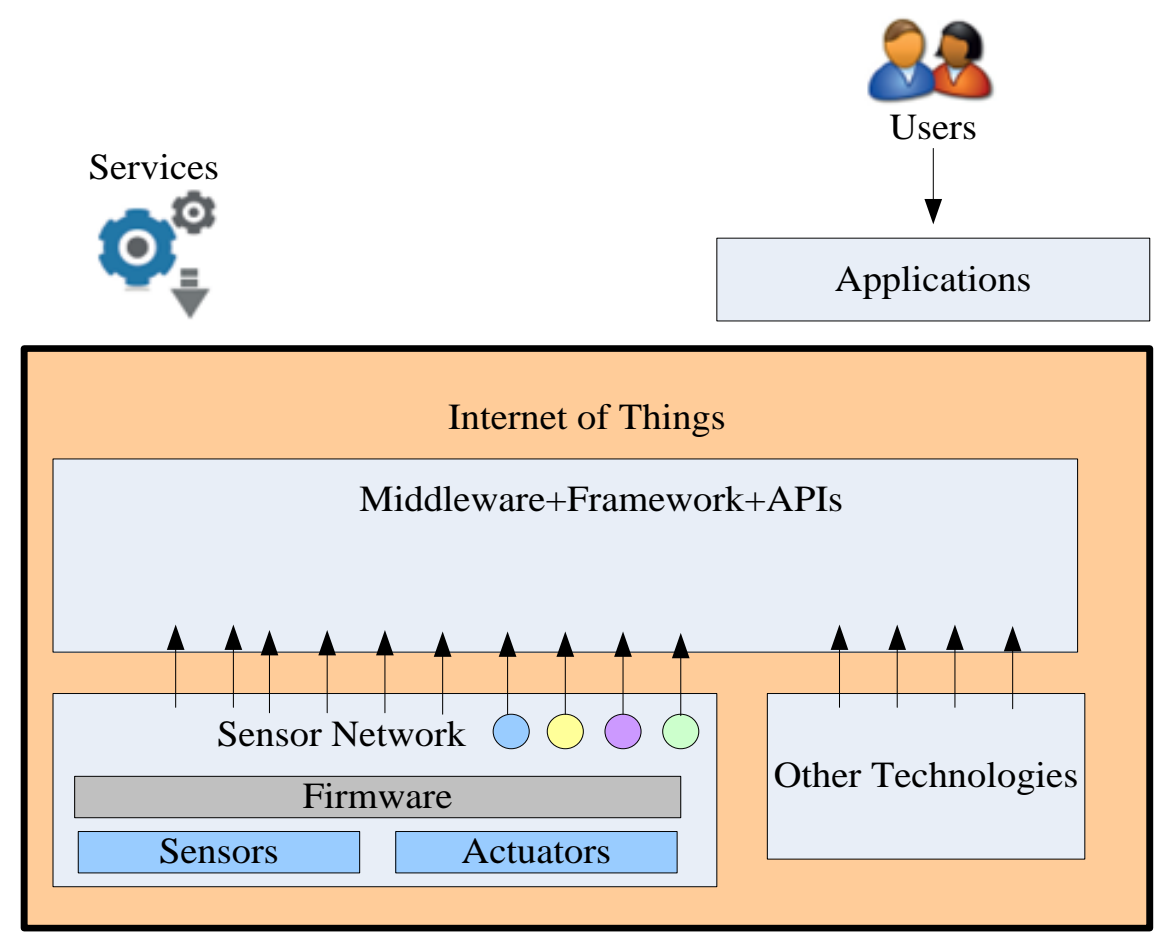

Fig.4. Relationship between IoT and Sensor Network 


\subsection{IoT Application Domains}

The IoT infrastructures enable various objects to be enabled and applicable in different types of domains such as industry, environment and society.

- Industry Focused Applications: Supply chain management, transportation and logistics, aerospace, aviation and automotive based application are considered as industry focused applications of IoT [9].

- Society Focused Applications: Telecommunication, medical technology, health care services, home, entertainment, smart building, Office, media, Ticketing related applications are considered as society oriented IoT applications.

- Environment focused Applications: Agriculture, breeding, recycling, and disaster alerting oriented applications are known as environment focused applications.

Various other 54 applications associated with development of a smart city, smart environment, smart metering, security, emergencies etc have been introduced in the study of [10].

\subsection{Challenges}

This section introduces various limitations associated with IoT infrastructure. The brief discussion is done over here and the following are the constraints associated with Internet of Things.

- Deployment of Sensor nodes In the future the sensor nodes deployment requires more attention in the field of cost of services, one of the biggest challenges in sensor technology is to maintain the sensor networks with zero effort. According to [11] many domestic projects were failed in execution because of various challenges associated with sensor node deployment. As it is not possible to replace the sensor batteries for their deployment in dynamic environment therefore another challenge arises which is low power sensor design in that concept a sensor do not require any type of battery change throughout the lifetime.

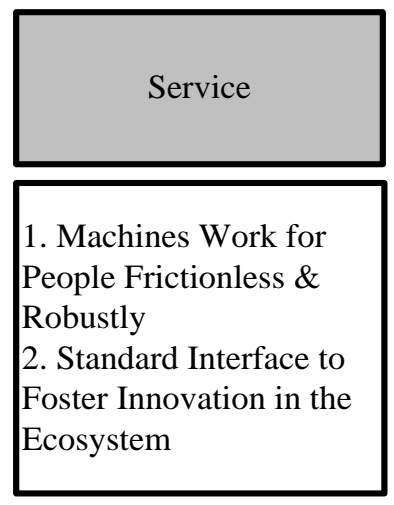

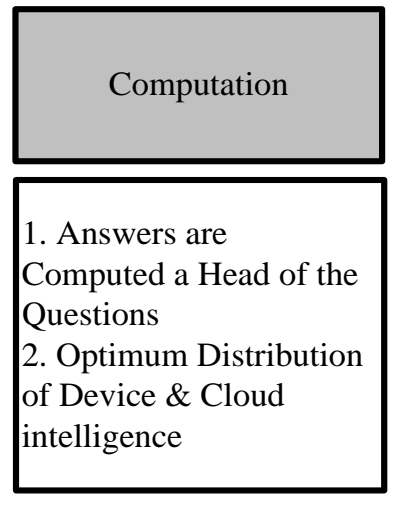

- Communication After the successful sensor node deployment, the collected data by sensors need to be sent with wireless communication services to the nearby base station. Even today the number of nodes interconnected with the internet is more than the number of human beings. In future various nodes will be connected through systems like $3 \mathrm{G} / 4 \mathrm{G}$ as well as $5 \mathrm{G}$ network environments. So the communication between the growing numbers of devices has become a huge challenge [12].

- Ease of Computation The connected devices which are deployed for generating huge amount of data needs more processing units. According to the data analysis of Cisco Networking Systems the existence of the number of objects on the internet surpassed the number of humans in the year of 2008 and 2009. In future the data generated by the various machines will impose key challenges in the field of data analysis and computing [13].

- Ease of Services After analyzing the pre-processed data machines or human being should take the proper actions. As in a search engine environment a machines provide various relevant searches and ask the users for the refining of search results thus in this case machines are using the users and this should not work in future as machines only works for humans for better designing of a IoT ecosystem which will give better services has become a crucial challenge. Fig 6 shows various major key challenges of Internet of things [14].

Apart from the above mentioned challenged in IoT, there exists other forms of issues too e.g. compliance requirements, third party request, security threats, data privacy, and access management. Fig.5 highlights some of the factors that can leverage the deployment challenges in IoT, which is majorly classified to 4 types viz. service challenge, computational challenge, communication challenge, and challenges residing in type of sensor to be used. Hence, any studies or research activities towards enhancing the performance of IoT should consider addressed the challenges discussed in this section for furnishing a better Quality-of-Services.

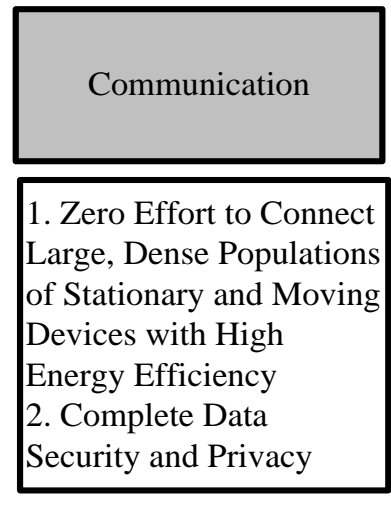

Fig.5. Major Challenges in Internet of Things

\section{RECENT SURVEY STUDIES}

This section discusses about the existing status of various review works which talks about the various technologies associated with internet of things. For better collection of information this proposed study refers various existing studies indexed in IEEE Explorer with the combination of search keyword "Internet of Things". It was found in the website IEEE digital library when the web server returns 510 journals and magazines, 4066 conference publications, 70 early access articles and 15 books in between 2010 to 2014. It is also observed that there are many Technical and Review papers which talks about various business transaction management systems of smart networks services. 
Table I. Summery of Existing Survey papers

\begin{tabular}{|c|c|c|c|}
\hline Authors & Year & Topic in focus & Inference \\
\hline Romer et al [15] & 2010 & Web Of Things & $\begin{array}{l}\text { Pros: Good Discussion About Domain. } \\
\text { Cons: Narrowed Survey About Prior } \\
\text { Techniques. }\end{array}$ \\
\hline Gluhak et al [16] & 2011 & Internet Of Things & $\begin{array}{l}\text { Pros: Explanation Of Very Few Prior } \\
\text { Techniques } \\
\text { Cons: No Comparison Between Prior Studies. }\end{array}$ \\
\hline Aziz et al [17] & 2013 & $\begin{array}{l}\text { Review Of Significant Topology } \\
\text { Control Techniques Of Internet Of } \\
\text { Things. }\end{array}$ & $\begin{array}{l}\text { Pros: Good Theoretical Discussion About } \\
\text { Domain. } \\
\text { Cons: Less Discussion About Prior Techniques. }\end{array}$ \\
\hline $\begin{array}{l}\text { Bellavista et al } \\
{[18]}\end{array}$ & 2013 & $\begin{array}{l}\text { Proposed An Original Solution For } \\
\text { Integrating The Iot Environment. }\end{array}$ & $\begin{array}{l}\text { Pros: Good Theory About Integrating The Iot } \\
\text { Environment. } \\
\text { Cons: Less Discussion About Existing Reviews. }\end{array}$ \\
\hline $\begin{array}{l}\text { Zhengguo et al } \\
\text { [19] }\end{array}$ & 2013 & $\begin{array}{l}\text { Technologies For Supporting Internet } \\
\text { Of Things }\end{array}$ & $\begin{array}{l}\text { Pros: Good Discussion About Technologies. } \\
\text { Cons: No Discussion About Prior Implemented } \\
\text { Techniques. }\end{array}$ \\
\hline $\begin{array}{l}\text { Abdelwahab et al } \\
\text { [20] }\end{array}$ & 2014 & $\begin{array}{l}\text { Survey Of Cloud Assisted Remote } \\
\text { Sensing (CARS) }\end{array}$ & $\begin{array}{l}\text { Pros: Good Discussion On Domain } \\
\text { Cons: Discussed Very Few Existing Prior } \\
\text { Techniques. }\end{array}$ \\
\hline $\begin{array}{l}\text { Zanella et al. } \\
\text { [21] }\end{array}$ & 2014 & $\begin{array}{l}\text { Enabling Technologies Of Internet Of } \\
\text { Things }\end{array}$ & $\begin{array}{l}\text { Pros: Good Theoretical Background. } \\
\text { Cons: Effective Contribution Not Found In } \\
\text { Terms Of Benchmark Studies And Comparative } \\
\text { Analysis. }\end{array}$ \\
\hline Chun et al [22] & 2014 & Internet Of Things & $\begin{array}{l}\text { Pros: Discussed Efficient Prior Techniques. } \\
\text { Cons: No Statistical Overview. }\end{array}$ \\
\hline Islam et al [23] & 2014 & $\begin{array}{l}\text { A Survey Of Evolution Of The Smart } \\
\text { Phones. }\end{array}$ & $\begin{array}{l}\text { Pros: Good Discussion On Domain. } \\
\text { Cons: Survey is repetitive in nature }\end{array}$ \\
\hline $\begin{array}{l}\text { Mometti et al } \\
{[24]}\end{array}$ & 2015 & Internet Of Things & $\begin{array}{l}\text { Pros: Good Analysis Of Prior Techniques. } \\
\text { Cons: Less Discussion About Domain. }\end{array}$ \\
\hline
\end{tabular}

\section{EXISTING TECHNIQUES}

It has been found that there are various techniques various IoT related techniques which have been discussed in journals of IEEE Digital library. The following Table II discusses few prior techniques of internet of things introduced by the researchers till date.
There are various techniques discussed till date for design and development of Internet-of-Things in past 5 years, however, we choose to select the most significant as well as contributory techniques of ten authors and performed analyzed deeply about the effectiveness of techniques.

Table II. Survey of Existing Techniques

\begin{tabular}{|l|l|l|l|}
\hline \multicolumn{1}{|c|}{ Authors } & \multicolumn{1}{|c|}{ Problems Identified } & \multicolumn{1}{c|}{ Proposed Architecture } & \multicolumn{1}{c|}{ Performance Parameters } \\
\hline Ortiz et al [25] & $\begin{array}{l}\text { The cluster between } \\
\text { Internet of Things (IoT) } \\
\text { and Social networks. }\end{array}$ & A generic S-IoT Architecture & Not Clearly Discussed \\
\hline $\begin{array}{l}\text { Debiao et al } \\
\text { [26] }\end{array}$ & $\begin{array}{l}\text { Security requirements of } \\
\text { RFID authentication. }\end{array}$ & $\begin{array}{l}\text { Elliptic Curve cryptography based } \\
\text { radio frequency identification } \\
\text { (RFID) Technology. }\end{array}$ & $\begin{array}{l}\text { Discussed in terms of the } \\
\text { proposed ECC based RFID } \\
\text { technique }\end{array}$ \\
\hline Oihui et al [27] & Enabling of general objects & $\begin{array}{l}\text { Operational framework of } \\
\text { cognitive internet of things (C- } \\
\text { IoT) }\end{array}$ & $\begin{array}{l}\text { Discusses the design of proper } \\
\text { performance metrics. }\end{array}$ \\
\hline Aijaz et al [28] & $\begin{array}{l}\text { Reliability of Cognitive } \\
\text { M2M communication }\end{array}$ & $\begin{array}{l}\text { Centralized cognitive MAC } \\
\text { protocol. }\end{array}$ & $\begin{array}{l}\text { Efficient Performance parameters } \\
\text { to ensure the protection of } \\
\text { proposed mechanism. }\end{array}$ \\
\hline $\begin{array}{l}\text { Daqiang et al } \\
\text { [29] }\end{array}$ & $\begin{array}{l}\text { Location based services for } \\
\text { Cellular phones }\end{array}$ & $\begin{array}{l}\text { Next Me } \\
\text { Experimental analysis } \\
\text { respect to performance parameters } \\
\text { show the effectiveness of the } \\
\text { proposed technique }\end{array}$ \\
\hline Nitti et al [30] & $\begin{array}{l}\text { Security in Internet of } \\
\text { Things }\end{array}$ & $\begin{array}{l}\text { Design of trust based } \\
\text { authentication model. }\end{array}$ & $\begin{array}{l}\text { Good Selection of performance } \\
\text { parameters to evaluate the } \\
\text { proposed model. }\end{array}$ \\
\hline Jiong et al [31] & Population Density & $\begin{array}{l}\text { Framework for realization of } \\
\text { smart cities. }\end{array}$ & $\begin{array}{l}\text { Narrowed discussion } \\
\text { Performance metrics. }\end{array}$ \\
\hline
\end{tabular}




\begin{tabular}{|c|c|c|c|}
\hline Hao et al [32] & $\begin{array}{l}\text { Expansion of Physical } \\
\text { objects in the field of IoT. }\end{array}$ & Data Clouds & $\begin{array}{l}\text { Good discussion of performance } \\
\text { metrics. }\end{array}$ \\
\hline $\begin{array}{l}\text { Cirani et al } \\
\text { [33] }\end{array}$ & $\begin{array}{l}\text { Resource } \quad \text { discovery } \\
\text { mechanism }\end{array}$ & $\begin{array}{l}\text { A scalable self-configuring peer to } \\
\text { peer based architecture. }\end{array}$ & $\begin{array}{l}\text { Performance parameter shows } \\
\text { how the proposed architecture is } \\
\text { more effective in nature. }\end{array}$ \\
\hline Li et al [34] & Security of IoT & 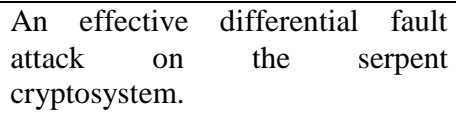 & $\begin{array}{l}\text { Good discussion about } \\
\text { Performance analysis with respect } \\
\text { to parameters. }\end{array}$ \\
\hline
\end{tabular}

\section{RESEARCH GAP}

After analyzing so many existing studies this section highlights some of the research gaps of the domain internet of things which are discussed as follows. The summery of existing survey papers published in between 2010 and 2015 are briefly discussed in Table I where it has been found that most of the papers added very limited contents about existing prior studies and discusses about the theoretical background of the problems. It has also been found that most of the papers indexed in the IEEE Explorer are repetitive in nature. The existing surveys hardly discusses about the prevention techniques for the constraints associated with the Internet of Things. Table II highlights various existing prior techniques where better classification of studies and scope towards future research direction are significantly missing. Most of prior techniques discussed in [29] [31] [32] are almost similar and no significant outcomes could be derived for the repetitive nature of implementation. From the information which have been gathered by Table I and Table II, it can be observed that majority of the existing studies do not discusses about the benchmark techniques thus it will be difficult for the future researchers to understand that which is the efficient technique to be adopted for continuing the research in the field of Internet of Things (IoT). Apart from the above discussed issues, the explored research gap in this area as discussed as follows:

- Less Emphasis on networking issues: In the modern computing era, every computing devices and network terminals are now represented as unique address. With the increasing attention of IoT application, the need of such unique address increases multifold even with the usage of IPv4 or IPv6 protocol. Another most complex issues in internet of things is non applicability of conventional internet protocol like TCP. Moreover, with the inclusion of heterogeneous devices for data acquisition, aggregation and processing, it becomes most difficult to address the issues of congestion. The existing congestion approach governed by TCP can streamline the traffic for end-to-end, but IoT requires only for short transmission of data.

- Complexity of inclusion of Routing: Routing is one of the significant operations being adopted for achieving effective communication standards in IoT. An effective implementation of routing could be done using source routing and hop-to-hop routing. However, any existing routing protocols are doesn't ensure reliability factor when it comes for heterogeneous network e.g. IoT owing to minimized delivery rate and significant delay.

- Security: Security is already a critical problems for all sorts of networking standards. The degree of security complexities differs in each type of networks. Hence, in IoT, it is quite difficult to identify the forms of attack and mitigate them using existing security approaches.

\section{CONCLUSION}

This proposed survey pay more attention on analyzing the various limitations associated with the existing survey studies highlighted in IEEE Explorer digital library whereas the detail analysis of the research gap on the domain of Internet of Things made this paper very attractive comparably other existing reviews. Table 2 discusses about various existing techniques and their research issues which could be valuable for the further research direction of Internet of Things. . It is believed that in future the Internet of Things will connect a huge amount of physical objects and will be able to process enormous amount of data as the society requires scalable, compatible and secure solution for management and complex architecture of Internet of things. The main objective of our future study is to create a framework for defining the future research clearly, which could give better resolution and scope to the future researchers.

\section{REFERENCES}

[1] Kleinwachter, W.; Almeida, V.A.F.2015. The Internet Governance Ecosystem and the Rainforest. Internet Computing, IEEE, Vol.19, No.2, pp.64-67

[2] Jindal, A.; Crutchfield, C.; Goel, S.; Kolluri, R.; Jain, R.2008.The mobile web is structurally different.INFOCOM Workshops 2008, IEEE, vol., no., pp.1,6, 13-18

[3] Baker, M.G.1994.Changing communication environments in MosquitoNet," Mobile Computing Systems and Applications, 1994. vol., no., pp.64-68

[4] Fox, M.S. 1993.Issues in enterprise modelling, Systems, Man and Cybernetics, 1993. vol., no., pp.86,92 vol.1, 1720

[5] Guillen, A.; Kia, R.N.; Sales, B.1993.An architecture for virtual circuit/QoS routing," Network Protocols, vol., no., pp.80-87

[6] Gershenfeld, Neil; Cohen, D.2006.Internet 0: Interdevice Internetworking - End-to-End Modulation for Embedded Networks.vol.22, no.5, pp.48-55

[7] Lord Wodehouse.1997.Building a better intranet Corporate Intranets.vol., no., pp.4/1-4/6

[8] McCluskey, P. 2002.A web-based graduate course on design-for-reliability of electronic systems. pp.15071509

[9] Wumnava, S.V.; Crosby, G.V.; Kapasi, A.2001.Adaptive network modeling scheme.vol., no., pp.109-113.

[10] Stern, Richard H. 1997. Shrink-wrap license restrictionspreempted. vol.17, no.1, pp.75-78.

[11] Nan Lin; Weihang Shi.2014. The research on Internet of things application architecture based on web. vol., no., pp.184-187. 
[12] Zhiyong Shi; Kui Liao; Shiping Yin; Qingbo Ou.2010.Design and implementation of the mobile internet of things based on td-scdma network.on, vol., no., pp.954,957, 17-19

[13] Yinghui Huang; Guanyu Li, 2010.A Semantic Analysis for Internet of Things. vol.1, no., pp.336,339, 11-12

[14] Coetzee, L.; Eksteen, J.2011.The Internet of Things promise for the future An introduction. vol., no., pp.1,9, 11-13 May 2011

[15] Romer, K.; Ostermaier, B.; Mattern, F.; Fahrmair, M.; Kellerer, W.2010.Real-Time Search for Real-World Entities: A Survey. vol.98, no.11, pp.1887-1902.

[16] Gluhak, A.; Krco, S.; Nati, M.; Pfisterer, D.; Mitton, N.; Razafindralambo, T.,2011.A survey on facilities for experimental internet of things research, vol.49, no.11, pp.58-67

[17] Aziz, A.A.; Sekercioglu, Y.A.; Fitzpatrick, P.; Ivanovich, M.2013.A Survey on Distributed Topology Control Techniques for Extending the Lifetime of Battery Powered Wireless Sensor Networks vol.15, no.1, pp.121144

[18] Bellavista, P.; Cardone, G.; Corradi, A.; Foschini, L.2013.Convergence of MANET and WSN in IoT Urban Scenarios.vol.13, no.10, pp.3558-3567

[19] Zhengguo Sheng; Shusen Yang; Yifan Yu; Vasilakos, A.; McCann, J.; Kin Leung.2013.A survey on the ietf protocol suite for the internet of things: standards, challenges, and opportunities pp.91-98.

[20]Abdelwahab, S.; Hamdaoui, B.; Guizani, M.; Rayes, A.2014.Enabling Smart Cloud Services Through Remote Sensing: An Internet of Everything Enabler.vol.1, no.3, pp.276-288.

[21] Zanella, A.; Bui, N.; Castellani, A.; Vangelista, L.; Zorzi, M.2014.Internet of Things for Smart Cities.vol.1, no.1, pp.22-32

[22] Chun-Wei Tsai; Chin-Feng Lai; Ming-Chao Chiang; Yang, L.T.2014. Data Mining for Internet of Things: A Survey.vol.16, no.1, pp.77-97

[23] Islam, N.; Want, R.2015.Smartphones: Past, Present, and Future.vol.13, no.4, pp.89-92.
[24] Bolic, Miodrag; Rostamian, Majed; Djuric, Petar M.2015.Proximity Detection with RFID: A Step Toward the Internet of Things. vol.14, no.2, pp.70-76.

[25] Ortiz, A.M.; Hussein, D.; Soochang Park; Han, S.N.; Crespi, N.2014.The Cluster Between Internet of Things and Social Networks: Review and Research Challenges.vol.1, no.3, pp.206-215

[26] Debiao He; Zeadally, S.2015.An Analysis of RFID Authentication Schemes for Internet of Things in Healthcare Environment Using Elliptic Curve Cryptography vol.2, no.1, pp.72-83

[27] Qihui Wu; Guoru Ding; Yuhua Xu; Shuo Feng; Zhiyong Du; Jinlong Wang; Keping Long .2014.Cognitive Internet of Things: A New Paradigm Beyond Connection.vol.1, no.2, pp.129-143

[28] Aijaz, A.; Aghvami, A.H.2015.Cognitive Machine-toMachine Communications for Internet-of-Things: A Protocol Stack Perspective. vol.2, no.2, pp.103-112.

[29] Daqiang Zhang; Shengjie Zhao; Yang, L.T.; Min Chen; Yunsheng Wang; Huazhong Liu.2015.NextMe: Localization Using Cellular Traces in Internet of Things.vol.11, no.2,pp.302-312

[30] Nitti，M.; Girau，R.; Atzori， L.2014.Trustworthiness Management in the Social Internet of Things.vol.26, no.5, pp.1253-1266.

[31] Jiong Jin; Gubbi, J.; Marusic, S.; Palaniswami, M.2014.An Information Framework for Creating a Smart City Through Internet of Things.vol.1, no.2, pp.112-121.

[32] Hao Yue; Linke Guo; Ruidong Li; Asaeda, H.; Yuguang Fang,2014.DataClouds: Enabling Community-Based Data-Centric Services Over the Internet of Things.vol.1, no. 5, pp.472-482

[33] Cirani, S.; Davoli, L.; Ferrari, G.; Leone, R.; Medagliani, P.; Picone, M.; Veltri, L.2014.A Scalable and SelfConfiguring Architecture for Service Discovery in the Internet of Things.vol.1, no.5, pp.508-521

[34] Li Wei; Tao Zhi; Gu Dawu; Sun Li; Qu Bo; Liu Zhiqiang; Liu Ya.2014.An effective differential fault analysis on the Serpent cryptosystem in the Internet of Things. vol.11, no.6, pp.129-139. 